\title{
Innovation at Nature Photonics
}

\author{
The option of 'double-blind' peer review, transfer consultations, shareable article links and online \\ Methods are all being introduced for authors.
}

February sees the introduction of a number of new features at Nature Photonics.

First, after listening to the desires of the scientific community, Nature Photonics, alongside Nature and other Nature branded journals, will shortly roll out the option of double-blind peer review for authors submitting papers. Indeed, several titles such as Nature Geoscience and Nature Climate Change have already conducted a successful 18 month experimental trial of this functionality.

Manuscripts undergoing doubleblind review will have their authorship kept anonymous to reviewers, just as the reviewers' identities are not disclosed to authors.

Our submission system will have a specific opt-in question, allowing authors to choose whether they wish their manuscript to be handled in a doubleblind manner or not. I should stress that this option is voluntary and purely at the discretion of authors and will not be enforced or encouraged by editors. Those opting in and wishing their manuscript to be handled in this manner should be sure to take all reasonable steps to ensure that their work is anonymous. Please note that it is ultimately the authors' responsibility to ensure that the manuscript is prepared in a way that does not reveal their identities. Editors can be consulted for advice if authors wish to have further guidance about how to do this effectively (see Box 1).

We are introducing this new option as various surveys and polls of reader opinion conducted by Nature Publishing Group have indicated that this is a functionality that would be welcomed by the majority of respondents. Although we of course cannot make a promise that reviewers will not guess the affiliations or identities of authors, our experience with authors trying to guess the identities of anonymous reviewers is that it's easy to get this wrong.

A second change that is being introduced at Nature Photonics is the use of online Methods - a well-established function that Nature has been using for some time.

Until now, the Methods section at the end of a Nature Photonics paper has been produced in identical forms in both

\section{Box 1 | Anonymity advice.}

- Do not include any author names or affiliations anywhere in the main paper or Supplementary Information files.

- Ensure that any acknowledgements, author contributions and author information are removed from the paper and placed in a cover letter instead.

- When referring to your own work within the paper, use neutral terminology and avoid phrases like "as we have shown previously".

- Do not include papers in the reference list that have not been accepted for publication yet.

- Ensure that any files submitted are not electronically tagged with authorship information revealing who created them - check the properties of the files within the software used to create them and edit as appropriate.

the print and online (PDF and HTML) versions and limited to just 800 words. The use of online Methods changes this. From February, all papers accepted for publication in Nature Photonics will be able to make use of the new Methods format, which allows for a significantly longer (up to 3,000 words) section that appears online only (PDF and HTML) and not in print. The online Methods section does not support the inclusion of any figures or tables. It does, however, support references that are listed at the end of the section, separate from the main paper's reference list. The numbering will naturally follow on from the last reference of the main paper and the number of references allowed in the section is flexible and not limited by the type of manuscript.

This development will allow authors to include more information that is relevant to how their experiments were conducted and analysed within the paper itself, rather than having to rely on the use of related Supplementary Information files. The change does not affect the inclusion of Supplementary Information files.

A third change is one that has already been recently introduced. We are now specifically asking authors during submission if they are happy for us to consult with the editors of other Nature branded journals in the case that Nature Photonics decides not to issue an offer to publish or revise a submission that it has received. Such consultations take place to determine if a sister journal would welcome the transfer of a paper with a view to making either an offer of immediate publication, revision or peer-review, as appropriate. Granting Nature Photonics permission to have this internal discussion does not in any way affect a manuscript's chances of publication in, or selection for peer review by, Nature Photonics itself. In contrast, it gives us a greater ability to help authors by attempting to find a suitable home for their manuscripts by securing an offer of publication in one of our journals.

A final new feature being introduced is shareable article links. Readers that have access to a Nature branded journal such as Nature Photonics (through either a personal subscription or an institutional arrangement) can now share a read-only version of a paper with anyone (including those that don't usually have access) using specially generated URL links. Simply use the share button within the full-text or ReadCube version of the paper and grab the generated link. This functionality is expected to be very useful for those referring to papers in blogs and authors wishing to update their biographies and publication lists with links that guarantee access to papers.

We hope that all of these new benefits and options prove useful.

\section{Correction}

In the Editorial 'A year to remember' (Nature Photonics 9, 1; 2015), the page number of the Review Article by Huanyang Chen and Lin Xu was incorrect and should have read 15 . This has now been corrected in the online versions after print 6 January 2015. 